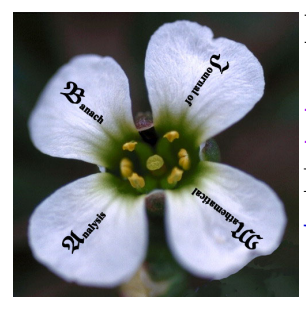

Banach J. Math. Anal. 8 (2014), no. 2, 146-155

Banach $\mathbf{J}_{\text {ournal of }} \mathbf{M}_{\text {athematical }} \mathbf{A}_{\text {nalysis }}$

ISSN: $1735-8787$ (electronic)

www.emis.de/journals/BJMA/

\title{
LUPAŞ-DURRMEYER OPERATORS BASED ON POLYA DISTRIBUTION
}

\author{
VIJAY GUPTA $^{1 *}$ AND THEMISTOCLES M. RASSIAS ${ }^{2}$
}

Communicated by S. S. Dragomir

\begin{abstract}
The generalization of the Bernstein polynomials based on Polya distribution is considered in the present article. Here, we introduce a mixed summation-integral type operators having Polya and Bernstein basis functions in summation and integration respectively. We establish some direct results which include an asymptotic formula, local and global approximation results for these operators in terms of modulus of continuity.
\end{abstract}

\section{INTRODUCTION AND PRELIMINARIES}

In the year 1968, Stancu [13] introduced a sequence of positive linear operators $P_{n}^{(\alpha)}: C[0,1] \rightarrow C[0,1]$, depending on a non-negative parameter $\alpha$ given by

$$
P_{n}^{(\alpha)}(f, x)=\sum_{k=0}^{n} f\left(\frac{k}{n}\right) p_{n, k}^{(\alpha)}(x),
$$

where $p_{n, k}^{(\alpha)}(x)$ is the Polya distribution with density function given by

$$
p_{n, k}^{(\alpha)}(x)=\left(\begin{array}{l}
n \\
k
\end{array}\right) \frac{\prod_{\nu=0}^{k-1}(x+\nu \alpha) \prod_{\mu=0}^{n-k-1}(1-x+\mu \alpha)}{\prod_{\lambda=0}^{n-1}(1+\lambda \alpha)}, x \in[0,1] .
$$

In case $\alpha=0$ these operators reduce to the classical Bernstein polynomials. For $\alpha=1 / n$ a special case of the operators (1.1) was considered by Lupaş and Lupaş

Date: Received: Nov. 10, 2013; Accepted: Dec. 16, 2013.

* Corresponding author.

2010 Mathematics Subject Classification. Primary 41A25; Secondary 47B33, 47B38.

Key words and phrases. Durrmeyer variant, Polya distribution, modulus of continuity, asymptotic formula, local and global approximation results. 
[4], which can be represented in an alternate form as

$$
P_{n}^{(1 / n)}(f, x)=\frac{2(n !)}{(2 n) !} \sum_{k=0}^{n}\left(\begin{array}{l}
n \\
k
\end{array}\right) f\left(\frac{k}{n}\right)(n x)_{k}(n-n x)_{n-k},
$$

where the rising factorial is given as $(x)_{n}=x(x+1)(x+2) \ldots(x+n-1)$. Recently Miclaus [12] established some approximation results for the operators (1.1) and for the case (1.2). In order to approximate Lebesgue integrable functions Durrmeyer [7] proposed the integral modification of usual Bernstein polynomials, which was later studied in different forms by Agrawal and Gupta [2], Abel et al. [1], GuptaIspir [10] and Gupta-Maheshwari [11] etc. We now propose the Durrmeyer type integral modification of the operators (1.2) as follows:

$$
D_{n}^{(1 / n)}(f, x)=(n+1) \sum_{k=0}^{n} p_{n, k}^{(1 / n)}(x) \int_{0}^{1} p_{n, k}(t) f(t) d t,
$$

where

$$
p_{n, k}^{(1 / n)}(x)=\frac{2(n !)}{(2 n) !}\left(\begin{array}{l}
n \\
k
\end{array}\right)(n x)_{k}(n-n x)_{n-k}
$$

and

$$
p_{n, k}(t)=\left(\begin{array}{l}
n \\
k
\end{array}\right) t^{k}(1-t)^{n-k}
$$

Some approximation properties related the present paper can be found in [8], [14] and in the recent books [3], [9]. In this paper, we consider the operator (1.3) and obtain a Voronovskaja type asymptotic formula and local and global direct estimates.

\section{Auxiliary Results}

We start this section with the following useful lemmas, which will be used in the sequel.

Lemma 2.1. (Miclaus [12]) For $e_{i}=t^{i}, i=0,1,2$ we have

$$
P_{n}^{(1 / n)}\left(e_{0}, x\right)=1, P_{n}^{(1 / n)}\left(e_{1}, x\right)=x
$$

and

$$
P_{n}^{(1 / n)}\left(e_{2}, x\right)=\frac{n x^{2}+2 x-x^{2}}{n+1}=x^{2}+\frac{2 x(1-x)}{n+1} .
$$

Lemma 2.2. For $e_{i}=t^{i}, i=0,1,2$ we have

$$
\begin{aligned}
& D_{n}^{(1 / n)}\left(e_{0}, x\right)=1, D_{n}^{(1 / n)}\left(e_{1}, x\right)=\frac{n x+1}{n+2} \\
& D_{n}^{(1 / n)}\left(e_{2}, x\right)=\frac{n^{3} x^{2}+5 n^{2} x-n^{2} x^{2}+3 n x+2 n+2}{(n+1)(n+2)(n+3)} .
\end{aligned}
$$

Proof. By simple computation, we have

$$
\int_{0}^{1} p_{n, k}(t) t^{r} d t=\frac{n !(k+r) !}{k !(n+r+1) !} .
$$


Obviously $D_{n}^{(1 / n)}\left(e_{0}, x\right)=1$ as $\int_{0}^{1} p_{n, k}(t) d t=\frac{1}{n+1}$. Next using (2.1) and applying Lemma 2.1, we have

$$
\begin{aligned}
D_{n}^{(1 / n)}\left(e_{1}, x\right) & =\sum_{k=0}^{n} p_{n, k}^{(1 / n)}(x) \frac{k+1}{n+2} \\
& =\frac{1}{n+2}\left[n P_{n}^{(1 / n)}\left(e_{1}, x\right)+P_{n}^{(1 / n)}\left(e_{0}, x\right)\right]=\frac{n x+1}{n+2} .
\end{aligned}
$$

Finally

$$
\begin{aligned}
D_{n}^{(1 / n)}\left(e_{2}, x\right) & =\sum_{k=0}^{n} p_{n, k}^{(1 / n)}(x) \frac{(k+1)(k+2)}{(n+2)(n+3)} \\
& =\frac{1}{(n+2)(n+3)}\left[n^{2} P_{n}^{(1 / n)}\left(e_{2}, x\right)+3 n P_{n}^{(1 / n)}\left(e_{1}, x\right)+2 P_{n}^{(1 / n)}\left(e_{0}, x\right)\right] \\
& =\frac{1}{(n+2)(n+3)}\left[\frac{n^{3} x^{2}+2 n^{2} x-n^{2} x^{2}}{n+1}+3 n x+2\right] \\
& =\frac{1}{(n+2)(n+3)}\left[\frac{n^{3} x^{2}+2 n^{2} x-n^{2} x^{2}+3 n^{2} x+3 n x+2 n+2}{n+1}\right] \\
& =\frac{n^{3} x^{2}+5 n^{2} x-n^{2} x^{2}+3 n x+2 n+2}{(n+1)(n+2)(n+3)} .
\end{aligned}
$$

Remark 2.3. By simple applications of Lemma 2.2, we have

$$
D_{n}^{(1 / n)}(t-x, x)=\frac{n x+1}{n+2}-x=\frac{1-2 x}{n+2}
$$

and

$$
\begin{aligned}
& D_{n}^{(1 / n)}\left((t-x)^{2}, x\right) \\
= & D_{n}^{(1 / n)}\left(t^{2}, x\right)-2 x D_{n}^{(1 / n)}(t, x)+x^{2} \\
= & \frac{n^{3} x^{2}+5 n^{2} x-n^{2} x^{2}+3 n x+2 n+2}{(n+1)(n+2)(n+3)}-2 x \frac{n x+1}{n+2}+x^{2} \\
= & \frac{\left(x-x^{2}\right)\left(3 n^{2}-5 n-6\right)+2(n+1)}{(n+1)(n+2)(n+3)} .
\end{aligned}
$$

Lemma 2.4. For $f \in C[0,1]$, we have $\left\|D_{n}^{(1 / n)}(f, x)\right\| \leq\|f\|$.

Proof. From the definition of operator and Lemma 2.2, we get

$$
\begin{aligned}
\left|D_{n}^{(1 / n)}(f, x)\right| & \leq(n+1) \sum_{k=0}^{n} p_{n, k}^{(1 / n)}(x) \int_{0}^{1} p_{n, k}(t)|f(t)| d t \\
& \leq\|f\| D_{n}^{(1 / n)}(1, x)=\|f\| .
\end{aligned}
$$


Lemma 2.5. For $n \in \mathbb{N}$, we have

$$
D_{n}^{(1 / n)}\left((t-x)^{2}, x\right) \leq \frac{3}{n+1} \delta_{n}^{2}(x)
$$

where $\delta_{n}^{2}(x)=\varphi^{2}(x)+\frac{1}{n+2}$, where $\varphi^{2}(x)=x(1-x)$.

Proof. By Lemma 2.2, we have

$$
\begin{aligned}
& D_{n}^{(1 / n)}\left((t-x)^{2}, x\right) \\
= & \frac{\left(x-x^{2}\right)\left(3 n^{2}-5 n-6\right)+2(n+1)}{(n+1)(n+2)(n+3)} \\
\leq & \frac{3}{n+1}\left[\varphi^{2}(x)+\frac{1}{n+2}\right]
\end{aligned}
$$

which is desired.

\section{Direct Estimates}

In this section, we first present the following asymptotic formula for the operators $D_{n}^{(1 / n)}(f, x)$.

Theorem 3.1. Let $f \in C[0,1]$ and If $f^{\prime \prime}$ exists at a point $x \in[0,1]$, then

$$
\lim _{n \rightarrow \infty} n\left[D_{n}^{(1 / n)}(f, x)-f(x)\right]=(1-2 x) f^{\prime}(x)+\frac{3 x(1-x)}{2} f^{\prime \prime}(x) .
$$

Proof. By Taylor's expansion of $f$, we have

$$
f(t)=f(x)+(t-x) f^{\prime}(x)+(t-x)^{2} \frac{f^{\prime \prime}(x)}{2}+\varepsilon(t, x)(t-x)^{2},
$$

where $\varepsilon(t, x) \rightarrow 0$ as $t \rightarrow x$. Applying $D_{n}^{(1 / n)}$ on above Taylor's expansion and using Remark 2.3, we have

$$
\begin{aligned}
D_{n}^{(1 / n)}(f, x)-f(x)= & f^{\prime}(x) D_{n}^{(1 / n)}((t-x), x)+\frac{1}{2} f^{\prime \prime}(x) D_{n}^{(1 / n)}\left((t-x)^{2}, x\right) \\
& \quad+D_{n}^{(1 / n)}\left(\varepsilon(t, x)(t-x)^{2}, x\right), \\
& \lim _{n \rightarrow \infty} n\left[D_{n}^{(1 / n)}(f ; x)-f(x)\right] \\
= & \lim _{n \rightarrow \infty} n f^{\prime}(x) D_{n}^{(1 / n)}((t-x), x)+\lim _{n \rightarrow \infty} n \frac{1}{2} f^{\prime \prime}(x) D_{n}^{(1 / n)}\left((t-x)^{2}, x\right) \\
& \quad+\lim _{n \rightarrow \infty} n D_{n}^{(1 / n)}\left(\varepsilon(t, x)(t-x)^{2}, x\right) \\
& \quad \lim _{n \rightarrow \infty} n\left[D_{n}^{(1 / n)}(f ; x)-f(x)\right] \\
= & (1-2 x) f^{\prime}(x)+\frac{3 x(1-x)}{2} f^{\prime \prime}(x)+\lim _{n \rightarrow \infty} n D_{n}^{(1 / n)}\left(\varepsilon(t, x)(t-x)^{2}, x\right) \\
= & (1-2 x) f^{\prime}(x)+\frac{3 x(1-x)}{2} f^{\prime \prime}(x)+F .
\end{aligned}
$$


In order to complete the proof, it is sufficient to show that $F=0$. By CauchySchwarz inequality, we have

$$
F=\lim _{n \rightarrow \infty} n D_{n}^{(1 / n)}\left(\varepsilon^{2}(t, x), x\right)^{1 / 2} D_{n}^{(1 / n)}\left((t-x)^{4}, x\right)^{1 / 2} .
$$

Furthermore, since $\varepsilon^{2}(x, x)=0$ and $\varepsilon^{2}(., x) \in C[0,1]$, it follows that

$$
\lim _{n \rightarrow \infty} n D_{n}^{(1 / n)}\left(\varepsilon^{2}(t, x), x\right)=0,
$$

uniformly with respect to $x \in[0,1]$. So from (3.1) and (3.2) we get

$$
\lim _{n \rightarrow \infty} n D_{n}^{(1 / n)}\left(\varepsilon^{2}(t, x), x\right)^{1 / 2} D_{n}^{(1 / n)}\left((t-x)^{4}, x\right)^{1 / 2}=0 .
$$

Thus, we have

$$
\lim _{n \rightarrow \infty} n\left[D_{n}^{(1 / n)}(f ; x)-f(x)\right]=(1-2 x) f^{\prime}(x)+\frac{3 x(1-x)}{2} f^{\prime \prime}(x),
$$

which completes the proof.

We begin by recalling the following $K$-functional:

$$
K_{2}(f, \delta)=\inf \left\{\|f-g\|+\delta\left\|g^{\prime \prime}\right\|: g \in W^{2}\right\} \quad(\delta>0),
$$

where $W^{2}=\left\{g \in C[0,1]: g^{\prime}, g^{\prime \prime} \in C[0,1]\right\}$ and $\|$.$\| is the uniform norm on$ $C[0,1]$. By [5], there exists a positive constant $C>0$ such that

$$
K_{2}(f, \delta) \leq C \omega_{2}(f, \sqrt{\delta})
$$

where the second order modulus of smoothness for $f \in C[0,1]$ is defined as

$$
\omega_{2}(f, \sqrt{\delta})=\sup _{0<h \leq \sqrt{\delta}} \sup _{x, x+2 h \in[0,1]}|f(x+2 h)-2 f(x+h)+f(x)| .
$$

We define the usual modulus of continuity for $f \in C[0,1]$ as

$$
\omega(f, \delta)=\sup _{0<h \leq \delta} \sup _{x, x+h \in[0,1]}|f(x+h)-f(x)| .
$$

Now we present direct local approximation theorem for the operator $D_{n}^{(1 / n)}(f, x)$.

Theorem 3.2. For the operators $D_{n}^{(1 / n)}$, there exists a constant $C>0$ such that

$$
\left|D_{n}^{(1 / n)}(f ; x)-f(x)\right| \leq C \omega_{2}\left(f,(n+1)^{-1} \delta_{n}(x)\right)+\omega\left(f,(n+2)^{-1}\right),
$$

where $f \in C[0,1], \delta_{n}(x)=\left[\varphi^{2}(x)+\frac{1}{n+1}\right]^{1 / 2}$ and $x \in[0,1]$.

Proof. We are introducing the auxiliary operators as follows

$$
\mathcal{D}_{n}^{(1 / n)}(f, x)=D_{n}^{(1 / n)}(f, x)+f(x)-f\left(\frac{n x+1}{n+2}\right) .
$$

Obviously by Lemma 2.2, we have

$$
\mathcal{D}_{n}^{(1 / n)}(1, x)=D_{n}^{(1 / n)}(1, x)=1
$$


and

$$
\mathcal{D}_{n}^{(1 / n)}(t, x)=D_{n}^{(1 / n)}(t, x)+x-\frac{n x+1}{n+2}=x .
$$

Let $g \in W^{2}$ and $t \in[0,1]$. By Taylor's expansion we have

$$
g(t)=g(x)+(t-x) g^{\prime}(x)+\int_{x}^{t}(t-u) g^{\prime \prime}(u) d u \text {. }
$$

Applying the operator $\mathcal{D}_{n}^{(1 / n)}$ to both sides of above equation, we get

$$
\begin{aligned}
\mathcal{D}_{n}^{(1 / n)}(g, x)(g, x)= & g(x)+\mathcal{D}_{n}^{(1 / n)}\left(\int_{x}^{t}(t-u) g^{\prime \prime}(u) d u\right) \\
= & g(x)+D_{n}^{(1 / n)}\left(\int_{x}^{t}(t-u) g^{\prime \prime}(u) d u, x\right) \\
& -\int_{x}^{\frac{n x+1}{n+2}}\left(\frac{n x+1}{n+2}-u\right) g^{\prime \prime}(u) d u .
\end{aligned}
$$

Hence

$$
\begin{aligned}
& \left|\mathcal{D}_{n}^{(1 / n)}(g, x)-g(x)\right| \\
& \leq \mathcal{D}_{n}^{(1 / n)}\left(\int_{x}^{t}|t-u|\left|g^{\prime \prime}(u)\right| d u, x\right) \\
& +\int_{x}^{\frac{n x+1}{n+2}}\left|\frac{n x+1}{n+2}-u\right|\left|g^{\prime \prime}(u)\right| d u \\
& \leq \mathcal{D}_{n}^{(1 / n)}\left((t-x)^{2}, x\right)\left\|g^{\prime \prime}\right\|+\left(\frac{n x+1}{n+2}-x\right)^{2}\left\|g^{\prime \prime}\right\| .
\end{aligned}
$$

On the other hand, from Lemma 2.5, we have

$$
\begin{aligned}
& D_{n}^{(1 / n)}\left((t-x)^{2}, x\right)+\left(\frac{1-2 x}{n+2}\right)^{2} \\
\leq & \frac{3}{n+1} \delta_{n}^{2}(x)+\left(\frac{1-2 x}{n+2}\right)^{2} \\
\leq & \frac{3}{n+1} \delta_{n}^{2}(x)+\frac{1}{(n+1)^{2}} \leq \frac{4}{n+1} \delta_{n}^{2}(x) .
\end{aligned}
$$

Thus, by (3.4) and (3.5), we have

$$
\left|\mathcal{D}_{n}^{(1 / n)}(g, x)-g(x)\right| \leq \frac{4}{n+1} \delta_{n}^{2}(x)\left\|g^{\prime \prime}\right\|,
$$

where $x \in[0,1]$. Furthermore, by Lemma 2.4, we get

$$
\begin{aligned}
\left|\mathcal{D}_{n}^{(1 / n)}(f, x)\right| & \leq\left|D_{n}^{(1 / n)}(f, x)\right|+|f(x)|+\left|f\left(\frac{n x+1}{n+2}\right)\right| \\
& \leq 3\|f\|,
\end{aligned}
$$

for all $f \in C[0,1]$. 
For $f \in C[0,1]$ and $g \in W^{2}$, using (3.6) and (3.7) we obtain

$$
\begin{aligned}
\left|D_{n}^{(1 / n)}(f, x)-f(x)\right|= & \left|\mathcal{D}_{n}^{(1 / n)}(f, x)-f(x)+f\left(\frac{n x+1}{n+2}\right)-f(x)\right| \\
\leq & \left|\mathcal{D}_{n}^{(1 / n)}(f-g, x)\right|+\left|\mathcal{D}_{n}^{(1 / n)}(g, x)-g(x)\right| \\
& +|g(x)-f(x)|+\left|f\left(\frac{n x+1}{n+2}\right)-f(x)\right| \\
\leq & 4\|f-g\|+\frac{4}{n+1} \delta_{n}^{2}(x)\left\|g^{\prime \prime}\right\|+\omega\left(f,\left|\frac{1-2 x}{(n+2)}\right|\right) .
\end{aligned}
$$

Taking infimum over all $g \in W^{2}$, we obtain

$$
\left|D_{n}^{(1 / n)}(f, x)-f(x)\right| \leq 4 K_{2}\left(f, \frac{1}{n+1} \delta_{n}^{2}(x)\right)+\omega\left(f,\left|\frac{1-2 x}{n+2}\right|\right)
$$

and by inequality (3.3), we get

$$
\left|D_{n}^{(1 / n)}(f ; x)-f(x)\right| \leq C \omega_{2}\left(f,(n+1)^{-1} \delta_{n}(x)\right)+\omega\left(f,(n+2)^{-1}\right)
$$

so proof is completed.

Let $f \in C[0,1]$ and $\varphi(x)=\sqrt{x(1-x)}, x \in[0,1]$. The second order DitzianTotik modulus of smoothness and corresponding $K$-functional are given by, respectively,

$$
\begin{aligned}
\omega_{2}^{\varphi}(f, \sqrt{\delta}) & =\sup _{0<h \leq \sqrt{\delta} x \pm h \varphi(x) \in[0,1]} \sup _{0}|f(x+h \varphi(x))-2 f(x)+f(x-h \varphi(x))|, \\
\bar{K}_{2, \varphi}(f, \delta) & =\inf \left\{\|f-g\|+\delta\left\|\varphi^{2} g^{\prime \prime}\right\|+\delta^{2}\left\|g^{\prime \prime}\right\|: g \in W^{2}(\varphi)\right\} \quad(\delta>0),
\end{aligned}
$$

where $W^{2}(\varphi)=\left\{g \in C[0,1]: g^{\prime} \in A C_{l o c}[0,1], \varphi^{2} g^{\prime \prime} \in C[0,1]\right\}$ and $g^{\prime} \in A C_{l o c}[0,1]$ means that $g$ is differentiable and $g^{\prime}$ is absolutely continuous on every closed interval $[a, b] \subset[0,1]$. We know from Theorem 1.3 .1 of [6] that there exists a positive constant $C>0$, such that

$$
\bar{K}_{2, \varphi}(f, \delta) \leq C \omega_{2}^{\varphi}(f, \sqrt{\delta}) .
$$

Also, the Ditzian-Totik modulus of first order is given by

$$
\vec{\omega}_{\psi}(f, \delta)=\sup _{0<h \leq \delta x \pm h \varphi(x) \in[0,1]} \sup _{0<f(x+h \psi(x))-f(x) \mid,}
$$

where $\psi$ is an admissible step-weight function on $[0,1]$.

Our last direct estimate is following global theorem in terms of weighted modulus of continuity.

Theorem 3.3. Let $f \in C[0,1]$. Then for $x \in[0,1]$, we have

$$
\left\|D_{n}^{(1 / n)} f-f\right\| \leq C \omega_{2}^{\varphi}\left(f,(n+1)^{-1 / 2}\right)+\vec{\omega}_{\psi}\left(f,(n+2)^{-1}\right),
$$

where $C>0$ is an absolute constant, $\psi(x)=1-2 x$ and $\varphi(x)=\sqrt{x(1-x)}$. 
Proof. Again we consider the auxiliary operators

$$
\mathcal{D}_{n}^{(1 / n)}(f, x)=D_{n}^{(1 / n)}(f, x)+f(x)-f\left(\frac{n x+1}{n+2}\right) .
$$

Using the definition of the operator $D_{n}^{(1 / n)}$ and Lemma 2.2, we obtained the inequality (3.5) in the proof of Theorem 3.2 as

$$
\begin{aligned}
& \left|\mathcal{D}_{n}^{(1 / n)}(g ; x)-g(x)\right| \\
& \leq D_{n}^{(1 / n)}\left(\int_{x}^{t}|t-u|\left|g^{\prime \prime}(u)\right| d u ; x\right) \\
& +\int_{x}^{\frac{n x+1}{n+2}}\left|\frac{n x+1}{n+2}-u\right|\left|g^{\prime \prime}(u)\right| d u .
\end{aligned}
$$

Moreover, $\delta_{n}^{2}$ is a concave function on $x \in[0,1]$, for $u=\lambda x+(1-\lambda) t, \lambda \in[0,1]$, we get

$$
\frac{|t-u|}{\delta_{n}^{2}(u)}=\frac{\lambda|t-x|}{\delta_{n}^{2}(\lambda x+(1-\lambda) t)} \leq \frac{\lambda|t-x|}{\lambda \delta_{n}^{2}(x)+(1-\lambda) \delta_{n}^{2}(t)} \leq \frac{|t-x|}{\delta_{n}^{2}(x)} .
$$

Thus, if we use this inequality in (3.9), we have

$$
\begin{aligned}
\left|\mathcal{D}_{n}^{(1 / n)}(g, x)-g(x)\right| & \leq D_{n}^{(1 / n)}\left(\int_{x}^{t} \frac{|t-u|}{\delta_{n}^{2}(u)} d u, x\right)\left\|\delta_{n}^{2} g^{\prime \prime}\right\| \\
& +\int_{x}^{\frac{n x+1}{n+2}} \frac{\left|\frac{n x+1}{n+2}-u\right|}{\delta_{n}^{2}(u)} d u\left\|\delta_{n}^{2} g^{\prime \prime}\right\| \\
& \leq \frac{1}{\delta_{n}^{2}(x)}\left\|\delta_{n}^{2} g^{\prime \prime}\right\|\left[D_{n}^{(1 / n)}\left((t-x)^{2}, x\right)+\left(\frac{1-2 x}{n+2}\right)^{2}\right] .
\end{aligned}
$$

By the inequality (3.5), we have

$$
\begin{aligned}
\left|\mathcal{D}_{n}^{(1 / n)}(g, x)-g(x)\right| & \leq \frac{3}{n+1}\left\|\delta_{n}^{2} g^{\prime \prime}\right\| \\
& \leq \frac{3}{n+1}\left(\left\|\varphi^{2} g^{\prime \prime}\right\|+\frac{1}{n+2}\left\|g^{\prime \prime}\right\|\right) .
\end{aligned}
$$

Using (3.7) and (3.10), we have for $f \in C[0,1]$,

$$
\begin{aligned}
\left|D_{n}^{(1 / n)}(f, x)-f(x)\right| \leq & \left|\mathcal{D}_{n}^{(1 / n)}(f-g, x)\right|+\left|\mathcal{D}_{n}^{(1 / n)}(g, x)-g(x)\right| \\
& +|g(x)-f(x)|+\left|f\left(\frac{n x+1}{n+2}\right)-f(x)\right| \\
\leq & 4\|f-g\|+\frac{4}{n+1}\left\|\varphi^{2} g^{\prime \prime}\right\|+\frac{4}{(n+1)^{2}}\left\|g^{\prime \prime}\right\| \\
& +\left|f\left(\frac{n x+1}{n+2}\right)-f(x)\right| .
\end{aligned}
$$


Taking infimum over all $g \in W^{2}$, we obtain

$$
\left|D_{n}^{(1 / n)}(f, x)-f(x)\right| \leq 4 \bar{K}_{2, \varphi}\left(f, \frac{1}{n+1}\right)+\left|f\left(\frac{n x+1}{n+2}\right)-f(x)\right| .
$$

On the other hand

$$
\begin{aligned}
& \left|f\left(\frac{n x+1}{n+2}\right)-f(x)\right| \\
& =\left|f\left(x+\psi(x) \cdot \frac{1-2 x}{(n+2) \psi(x)}\right)-f(x)\right| \\
& \leq \sup _{t, t+\psi(t) \frac{1-2 x}{(n+2) \psi(x)} \in[0,1]}\left|f\left(t+\psi(t) \frac{1-2 x}{(n+2) \psi(x)}\right)-f(t)\right| \\
& \leq \vec{\omega}_{\psi}\left(f, \frac{|1-2 x|}{(n+2) \psi(x)}\right) \\
& =\vec{\omega}_{\psi}\left(f, \frac{1}{(n+2)}\right) \text {. }
\end{aligned}
$$

Therefore, from (3.8) and (3.11) we obtain

$$
\left\|D_{n}^{(1 / n)} f-f\right\| \leq C \omega_{2}^{\varphi}\left(f,(n+1)^{-1 / 2}\right)+\vec{\omega}_{\psi}\left(f,(n+2)^{-1}\right),
$$

which is the desired result.

\section{REFERENCES}

1. U. Abel, V. Gupta and R.N. Mohapatra, Local approximation by a variant of BernsteinDurrmeyer operators, Nonlinear Anal. 68 (11) (2008), 3372-3381.

2. P.N. Agrawal and V. Gupta, Simultaneous approximation by linear combination of modified Bernstein polynomials, Bull. Greek Math. Soc. 39 (1989), 29-43.

3. A. Aral, V. Gupta and R.P. Agarwal, Applications of $q$ Calculus in Operator Theory, Springer, New York, 2013.

4. L. Lupaş and A. Lupas, Polynomials of binomial type and approximation operators, Studia Univ. Babes-Bolyai, Mathematica 32 no. 4 (1987), 61-69.

5. R.A. DeVore ang G.G. Lorentz, Constructive Approximation, Grundlehren der Mathematischen Wissenschaften, Band 303, Springer-Verlag, Berlin, Heidelberg, New York and London, 1993.

6. Z. Ditzian and V. Totik, Moduli of smoothness, Springer Series in Computational Mathematics, 9. Springer-Verlag, New York, 1987.

7. J.L. Durrmeyer, Une formule d' inversion de la Transformee Laplace, Applications a la Theorie des Moments, These de 3e Cycle, Faculte des Sciences de I' Universite de Paris, 1967.

8. S.M. Farsani, On the boundedness and compactness of a certain integral operator, Banach J. Math. Anal. 7 no. 2 (2013), 86-102.

9. V. Gupta and R.P. Agarwal, Convergence Estimates in Approximation Theory, Springer, New York, 2014.

10. V. Gupta and N. Ispir, On simultaneous approximation for some modified Bernstein-type operators, Int. J. Math. Math. Sci. 71 (2004), 3951-3958.

11. V. Gupta and P. Maheshwari, Bézier variant of a new Durrmeyer type operators, Riv. Mat. Univ. Parma (N.S.) 7 no. 2 (2003), 9-21.

12. D. Miclăuş, The revision of some results for Bernstein Stancu type operators, Carpathian J. Math. 28 (2) (2012), 289-300. 
13. D.D. Stancu, Approximation of functions by a new class of linear polynomial operators, Rev. Roum. Math. Pures Appl. 13 (1968), 1173-1194.

14. G.V. Milovanović, D.S. Mitrinović and Th. M. Rassias, Topics in polynomials: extremal problems, inequalities, zeros, World Scientific Publishing Co., Inc., River Edge, NJ, 1994.

1 Department of Mathematics, Netaji Subhas Institute of Technology, Sector 3 Dwarka, New Delhi 110078, India.

E-mail address: vijaygupta2001@hotmail.com

2 Department of Mathematics, National Technical University of Athens, Zografou Campus, 157 80, Athens, Greece.

E-mail address: trassias@math.ntua.gr 